\title{
Cerebroplacental ratio in gestational hypertension and its impact on neonatal weight
}

\begin{abstract}
Background: Pregnancy a physiological process that involves changes in vascular system responsiveness that affects the placental development in direct and indirect methods by hormonal and other signals that are critical in normal blood flow rates within the fetal system. Predicting fetal outcome using sonographic indices is considered a noninvasive valuable protocol to manage cases with hypertensive disorders with pregnancy. Fetal weight is one of the critical issues faced by obstetricians and perinatologists.
\end{abstract}

Aim of the study: to determine the value of cerebro placental ratio in predictability of neonatal weight in cases of gestational hypertension.

Patients and methods: A prospective observational cross sectional study that included 100 pregnant women, 50 ladies had gestational hypertension and 50 women as normal control group attending the antenatal care clinic of Algezeera hospital. Ultrasonographic assessment was carried out with evaluation of routine fetal biometry in addition to Middle cerebral artery (MCA), Umbilical artery (UA) Doppler indices.

Results: UA PI, MCA PI, CPR, Fetal weight and birth weight were statistically significantly higher among the control research group in comparison to the hypertension with pregnancy research group ( $\mathrm{p}$ values $<0.001)$.

Conclusion: Cerebroplacental ratio is a promising ultrasonographic marker for fetal and neonatal weight that could aid in prediction of neonatal wellbeing and clinical prognosis of hypertensive cases in pregnancy.
Volume II Issue 5 - 2020

\author{
Mahmoud Alalfy,' Ahmed Okasha,' Omar \\ Abdalfatah,' E Eltaieb² \\ 'Reproductive health and family planning department, National \\ Research Centre, Egypt \\ ${ }^{2}$ Lecturer in Obstetrics and Gynecology, Ainshams University, \\ Cairo, Egypt
}

\begin{abstract}
Correspondence: Mahmoud Alalfy, PhD. Reproductive health and family planning department, National Research Centre, Dokki, P.O:I2622, Egypt, Affiliation ID: 600I46I8 and Consultant OB/Gyn, Aljazeerah Hospital, Egypt, Tel +2010026 I I058, Emailmahmoualalfy@ymail.com
\end{abstract}

Received: September 23, 2020 | Published: September 30, 2020

\section{Introduction}

Pregnancy a physiological process that involves changes in vascular system responsiveness that affects the placental development in direct and indirect methods by hormonal and other signals that are critical in normal blood flow rates within the fetal system. Blood flow rates within the fetal system would affect the fetal development and growth rates. ${ }^{1,2}$

Hypertensive diseases during pregnancy would have a pathophysiological impact on placental development and consequently could affect the fetal blood flow rates causing adaptive changes such as brain sparing effect that could affect the fetal weight and wellbeing., ${ }^{3,4}$

Umbilical, middle cerebral pulsatility index and cerebroplacental ratios are induces that could be used in analysis of fetal wellbeing status. Hypertensive issues during pregnancy could definitely have an impact on those indices in a manner that could reflect the pathophysiological process affecting the blood flow rates within the fetal vascular system. ${ }^{5,7}$

Predicting fetal outcome using ultrasonographic indices is considered a noninvasive valuable protocol to manage cases with hypertensive disorders with pregnancy. Fetal weight is one of the critical issues faced by obstetricians and perinatologists since it determines the capability of normal extra uterine existence without short or long term clinical complications. ${ }^{8}$

Aim: To determine the value of cerebroplacental ratio in predictability of neonatal weight in cases of gestational hypertension.

\section{Patients and methods}

A prospective observational cross sectional study that included 100 pregnant women, 50 ladies had gestational hypertension and 50 women as normal control group attending the antenatal care clinic of Algezeera hospital in the period from February 2019 till January 2020. Ultrasonographic assessment was carried out with evaluation of routine fetal biometry in addition to Middle cerebral artery (MCA), Umbilical artery (UA) Doppler indices. Inclusion criteria of both cases and controls involved the following: no active labor at ultra ultrasonographic examination time, no fetal anomalies, multiple pregnancy, and precise dating of cases.

Detailed history taking with physical examination and categorization of cases according to clinical status was undergone besides full ultrasonographic assessment was carried out with evaluation of routine fetal biometry besides MCA, UA Doppler indices.

\section{Statistical methods}

Data were coded and entered using the statistical package for the Social Sciences (SPSS) version 26 (IBM Corp., Armonk, NY, USA). Data was summarized using mean and standard deviation for quantitative variables and frequencies (number of cases) and relative frequencies (percentages) for categorical variables. Comparisons between groups were done using unpaired t test. ${ }^{9}$ For comparing categorical data, Chi square $\left(\chi^{2}\right)$ test was performed. Exact test was used instead when the expected frequency is less than $5 .{ }^{10} \mathrm{P}$-values less than 0.05 were considered as statistically significant. 


\section{Results}

Table 1 reveals and displays that there was statistical significant difference between both research groups regarding parity was statistically significantly higher among control research group $\mathrm{P}$ value $=0.017$, wereas there was no statistical significant difference between both research groups as regards the miscarriage and mode of delivery $(\mathrm{p}$ values $=0.069,0.689$ consecutively) (Figure 1$)$.

Table I Comparison between control and hypertension with pregnancy research groups

\begin{tabular}{|c|c|c|c|c|c|c|}
\hline & & \multicolumn{5}{|l|}{ Group } \\
\hline & & \multicolumn{2}{|c|}{ Control group } & \multicolumn{2}{|c|}{ Hypertension pregnancy } & \multirow{2}{*}{$P$ value } \\
\hline & & Count & $\%$ & Count & $\%$ & \\
\hline \multirow{6}{*}{ Parity } & PG & 4 & $8.00 \%$ & 8 & $16.00 \%$ & \multirow{6}{*}{0.017} \\
\hline & 1 & 2 & $4.00 \%$ & 10 & $20.00 \%$ & \\
\hline & 2 & 17 & $34.00 \%$ & 17 & $34.00 \%$ & \\
\hline & 3 & 19 & $38.00 \%$ & 14 & $28.00 \%$ & \\
\hline & 4 & 7 & $14.00 \%$ & 1 & $2.00 \%$ & \\
\hline & 5 & 1 & $2.00 \%$ & 0 & $0.00 \%$ & \\
\hline \multirow{5}{*}{ Miscarriage } & 0 & 17 & $34.00 \%$ & 19 & $38.00 \%$ & \multirow{5}{*}{0.069} \\
\hline & 1 & 12 & $24.00 \%$ & 14 & $28.00 \%$ & \\
\hline & 2 & 12 & $24.00 \%$ & 15 & $30.00 \%$ & \\
\hline & 3 & 9 & $18.00 \%$ & 1 & $2.00 \%$ & \\
\hline & 12 & 0 & $0.00 \%$ & 1 & $2.00 \%$ & \\
\hline \multirow{2}{*}{ MOD } & CS & 24 & $48.00 \%$ & 26 & $52.00 \%$ & \multirow{2}{*}{0.689} \\
\hline & NVD & 26 & $52.00 \%$ & 24 & $48.00 \%$ & \\
\hline
\end{tabular}

\section{घ control group $\quad$ a hypertension pregnancy group}

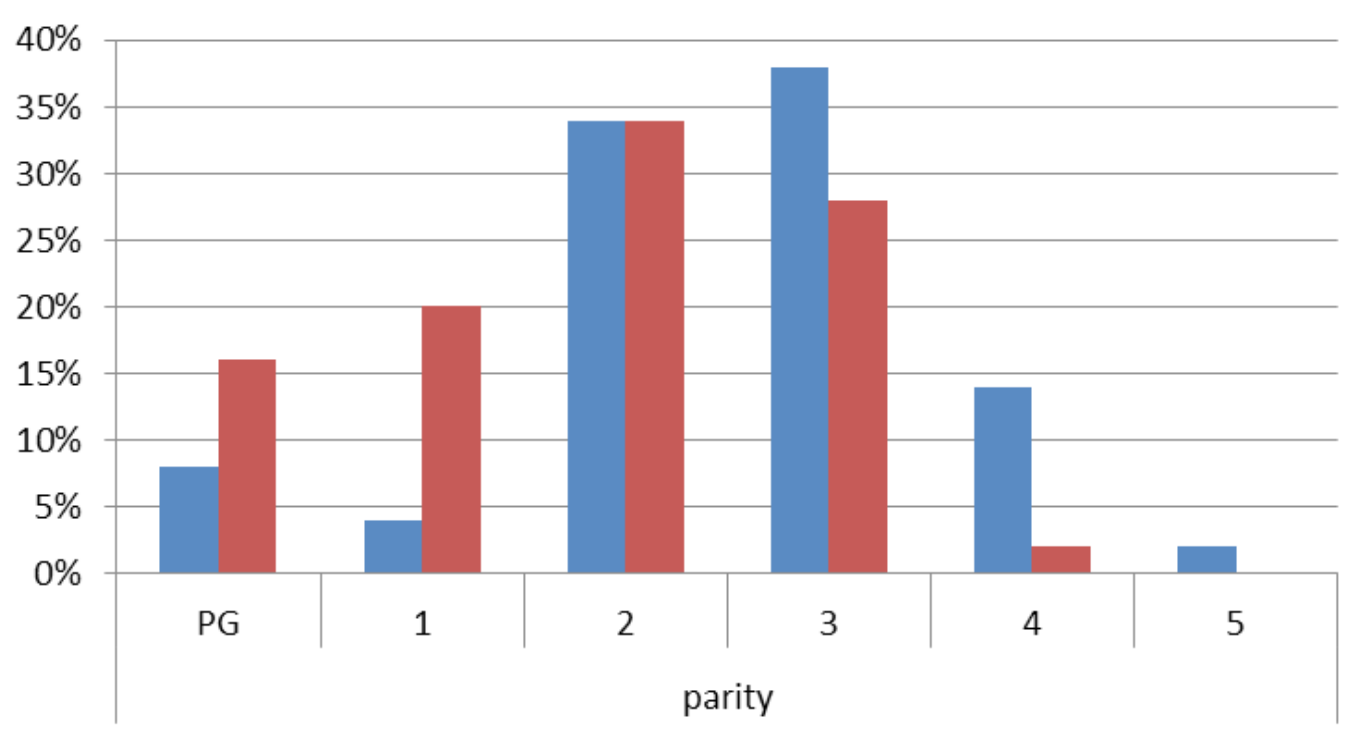

Figure I Comparison of parity among the investigated research groups.

Table 2, Figures 2-6 reveals and displays that there was no statistical significant difference between both research groups as regards age, BMI and GA ( $\mathrm{P}$ values $=0.500,0.838,0.382$ consecutively) whereas UA PI,MCA PI, CPR, Fetal weight and birth weight were statistically significantly higher among the control research group in comparison to the hypertension with pregnancy research group ( $\mathrm{p}$ values $<0.001$ ).

Table 3 reveals that there was statistical significant difference as regards the mean parity between the control and hypertensive research groups (Figure 7). 
Table 2 Comparison between investigated research groups as regards demographic, ultrasonographic and fetal indices

\begin{tabular}{|c|c|c|c|c|c|c|c|c|c|}
\hline & \multicolumn{9}{|l|}{ Group } \\
\hline & \multicolumn{4}{|c|}{ Control group } & \multicolumn{4}{|c|}{ Hypertension pregnancy } & \multirow{2}{*}{$P$ value } \\
\hline & Mean & SD & Minimum & Maximum & Mean & SD & Minimum & Maximum & \\
\hline Age & 30.14 & 5.65 & 21 & 39 & 29.4 & 5.26 & 21 & 39 & 0.5 \\
\hline BMI & 27.2 & 1.5 & 24.5 & 29.5 & 27.26 & 1.43 & 25 & 29.5 & 0.838 \\
\hline GA & 37.2 & 1.87 & 33 & 40 & 37.48 & 1.25 & 35 & 40 & 0.382 \\
\hline UA PI & 0.92 & 0.1 & 0.68 & 1.2 & 1.13 & 0.18 & 0.88 & 1.5 & $<0.001$ \\
\hline MCA PI & 1.4 & 0.21 & 0.31 & 1.8 & 1.25 & 0.13 & 1 & 1.6 & $<0.001$ \\
\hline CPR & 1.53 & 0.25 & 0.4 & 2.3 & 1.13 & 0.19 & 0.9 & 1.5 & $<0.001$ \\
\hline Fetal weight & 3005 & 339.59 & 2250 & 3800 & 2257.4 & 373.67 & 1800 & 3000 & $<0.001$ \\
\hline Birth Weight & 3192.3 & 336 & 2500 & 4000 & 2433.1 & 386.43 & 1850 & 3200 & $<0.001$ \\
\hline
\end{tabular}

Table 3 Parity mean among control and hypertension with pregnancy research groups

\begin{tabular}{|c|c|c|c|c|c|c|c|c|c|}
\hline & \multicolumn{9}{|l|}{ Group } \\
\hline & \multicolumn{4}{|c|}{ Control group } & \multicolumn{4}{|c|}{ Hypertension pregnancy } & \multirow{2}{*}{$P$ value } \\
\hline & Mean & SD & Minimum & Maximum & Mean & SD & Minimum & Maximum & \\
\hline Parity & 2.52 & 1.111 & 0 & 5 & 1.8 & 1.088 & 0 & 4 & 0.001 \\
\hline
\end{tabular}

\section{UA PI}

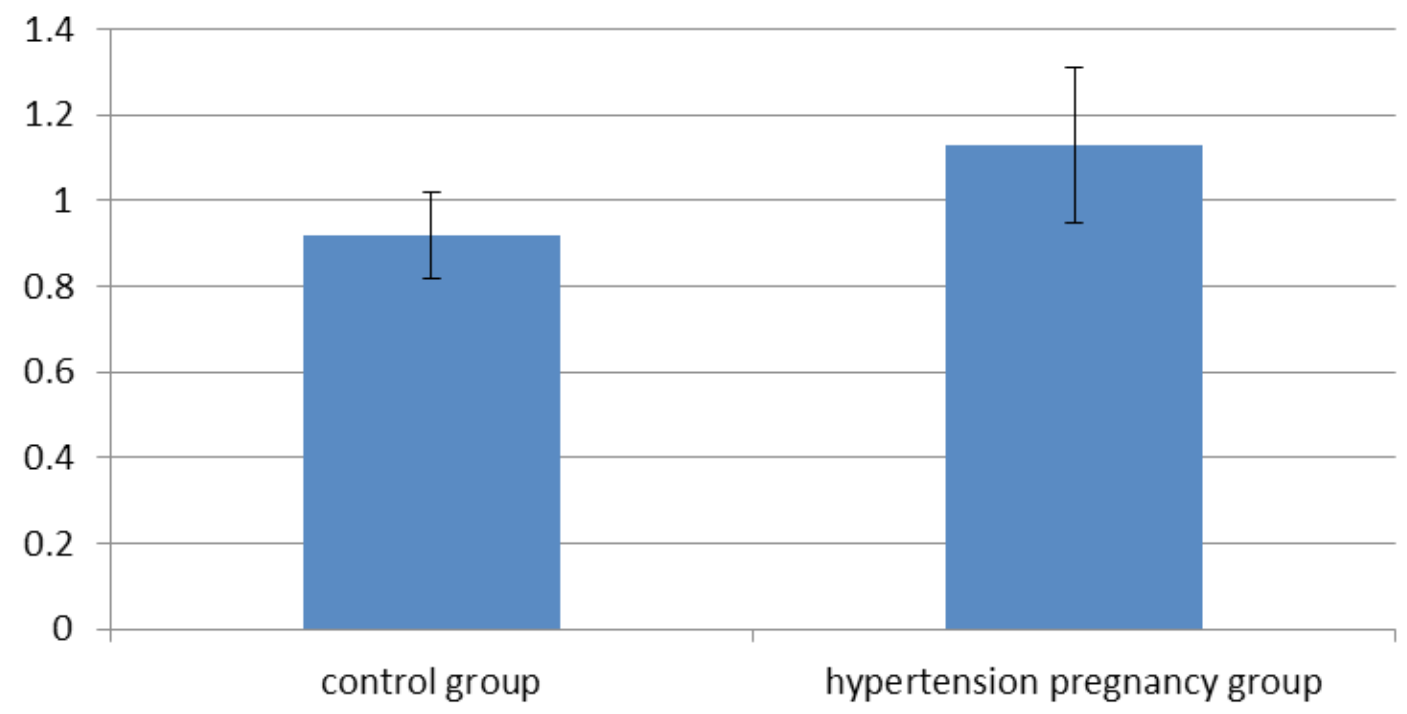

Figure 2 Comparison of umbilical artery PI between control and hypertension research groups. 


\section{MCA PI}

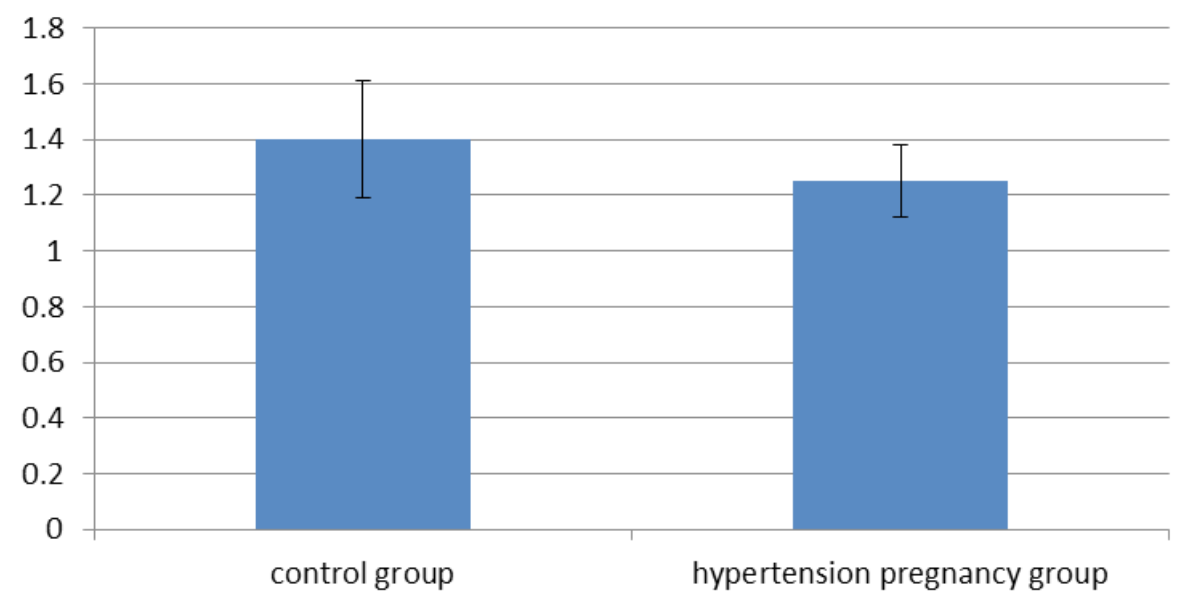

Figure 3 Comparison of middle cerebral artery PI between control and hypertension research groups.

\section{CPR}

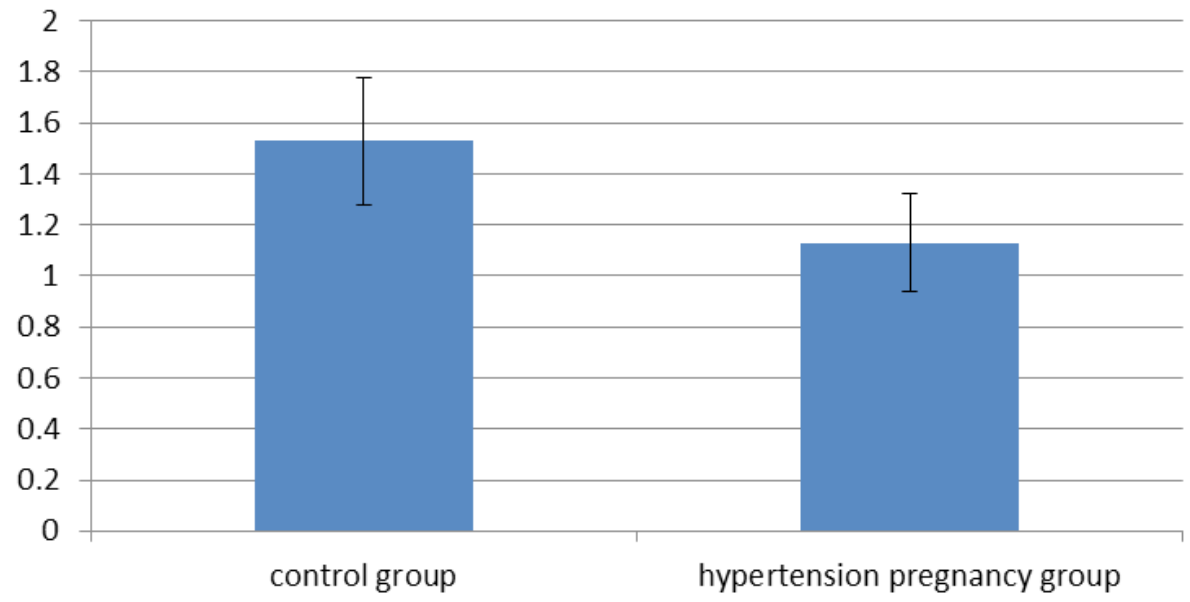

Figure 4 Comparison of cerebro placental ratio between control and hypertension research groups.

\section{fetal weight}

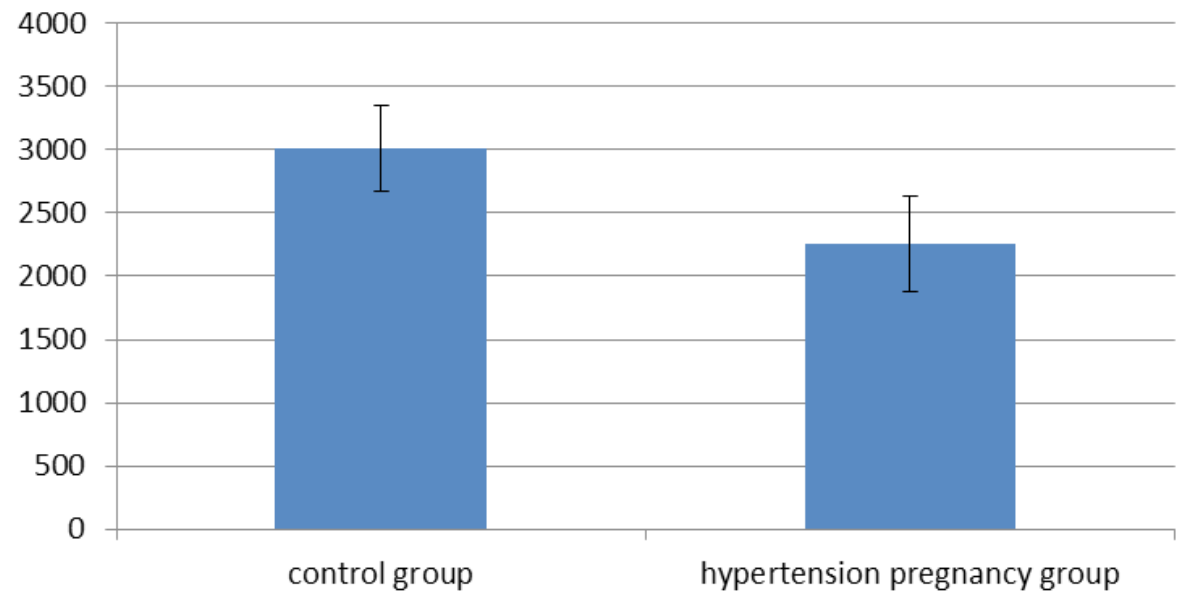

Figure 5 Comparison of fetal weight between control and hypertension research groups. 


\section{birth weight}

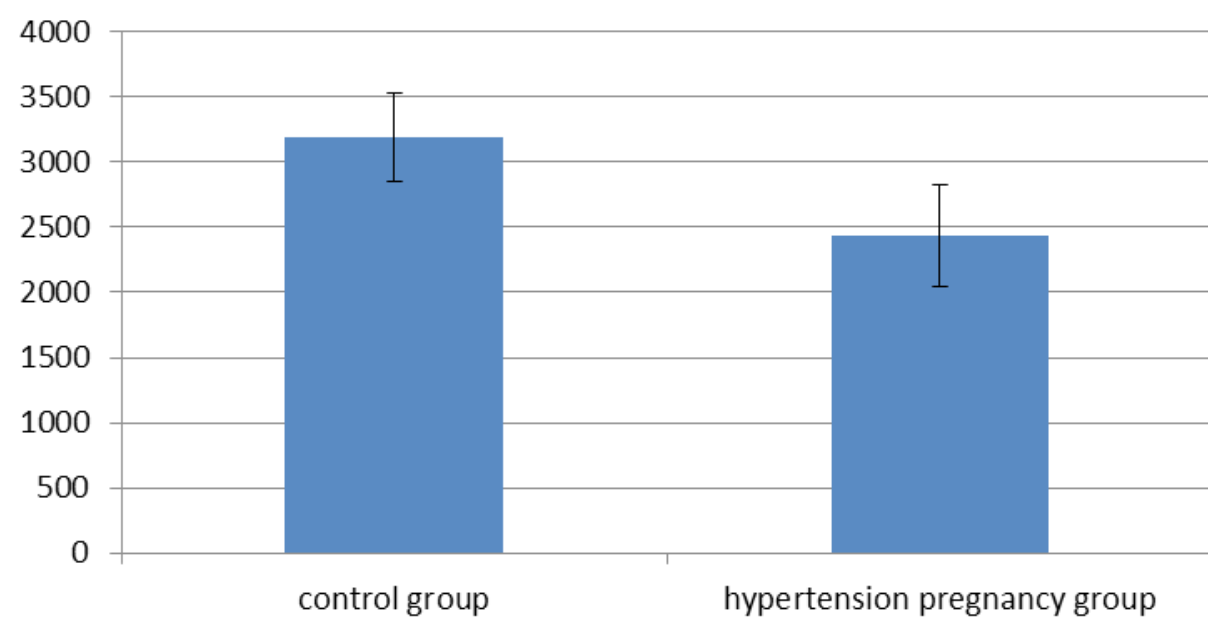

Figure 6 Comparison of birth weight between control and hypertension research groups.

\section{parity}

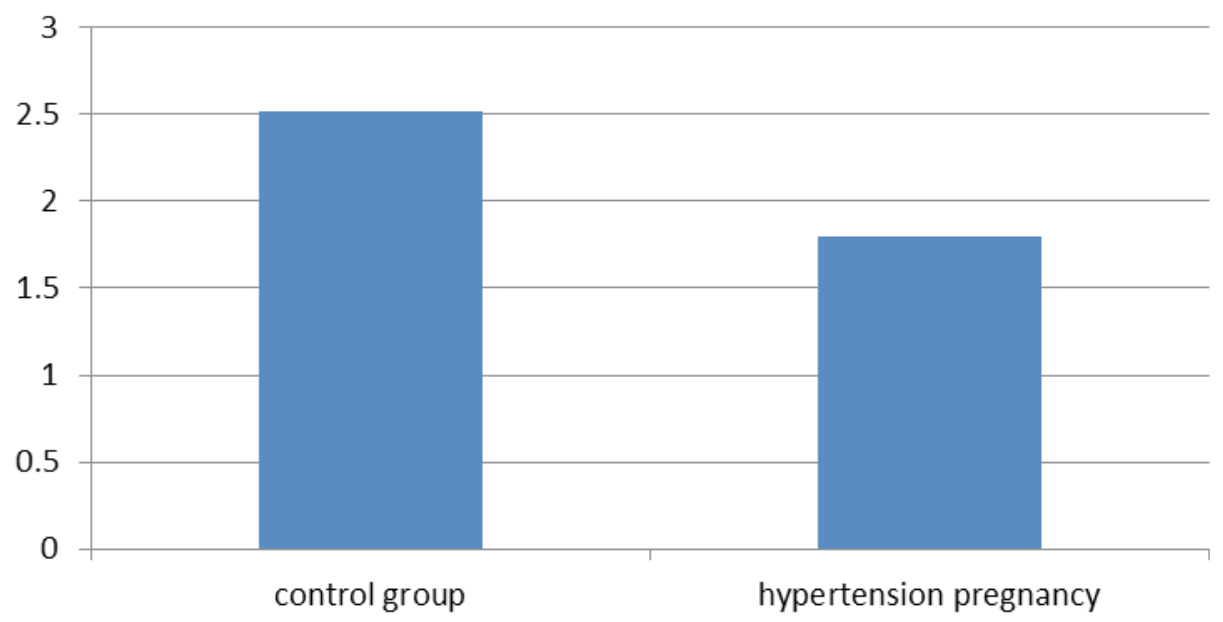

Figure 7 Parity mean among control and hypertensive research groups.

\section{Discussion}

Hypertensive issues is a common clinical scenario facing obstetricians all over the globe hypertension developing during gestation or preexisting before pregnancy could seriously affect the fetal growth pattern by influencing the pattern of placental vascular development and could affect the placental process of aging at cellular and various vascular fetal indices are affected as a result. ${ }^{11}$

Previous research groups of investigators investigated the fetal vascular indices as a noninvasive method to predict the fetal wellbeing and its impact on fetal and neonatal weight. Umilical artery one of the crucial vascular indices reflects the main artery vascular flow that is affected by angiogenic factors released during pregnancy .Middle cerebral artery PI was observed from previous research studies to detect and reflect the brain sparing effect that could be an adaptive protective mechanism in casee of reduced placental blood flow from the umbilical vascular system. In an interesting manner similar to the current research study effort the cerebroplacental ratio was observed to be a possibly valuable tool in assessment of fetal vascular system status particular in correkation to fe6tal and neonatal weight. ${ }^{1,3}$

In harmony with the current research study findings it was observed by a prior research group of investigators that out of the fifty pregnancy induced hypertension study subjects investigated, thirty-six revealed statistical significant positive Doppler indices as regards the umbilical ,middle cerebral and uterine artery indices however the current research didn't investigate the uterine artery indices. Furthermore in great similarity to the current study finding a previous investigators revealed that the cerebroplacental ratio i.e. MCA/Umbilical artery PI] was $<1$ in $69 \%$ of investigated cases. ${ }^{4,7}$

The current research study interestingly have investigated 100 research study subjects 50 cases (hypertension with pregnancy), 50 control cases both in comparative manner have been analyzed revealing the following there was statistical significant difference between both research groups regarding parity was statistically significantly higher among control research group $\mathrm{P}$ value $=0.017$ those research findings 
could be justified by the fact that Primigravida cases are more liable to development of preeclampsia and hypertensive issues particularly in young age groups. ${ }^{2}$

Whereas there was no statistical significant difference between both research groups as regards the miscarriage and mode of delivery ( $p$ values $=0.069,0.689$ consecutively). Furthermore the current research study have revealed that there was no statistical significant difference between both research groups as regards age, BMI and GA (P values $=0.500,0.838,0.382$ consecutively) those research findings are contradicting with previous research efforts as regards the BMI since it was clearly observed that cases having high BMI indices are more lible to hypertensive issues during gestation besides other medical conditions that could be justified by the fact that the current study had a small sample size and all cases were under 30 as regard the BMI .However UA PI,MCA PI,CPR, Fetal weight and birth weight were statistically significantly higher among the control research group in comparison to the hypertension with pregnancy research group ( $p$ values $<0.001$ ).those research findings have shown great harmony and similarity with previous research studies that investigated the same fetal vascular indices with and without placental biomarkers in which it was observed that fetal and neonatal weight were statistically significantly higher among normal cases in comparison to cases that developed preeclampsia and other hypertensive issues during pregnancy interestingly those research findings could be justified by the fact that the vascular dialogue ta hormonal and cellular levels is greatly inpacted by the pathophysiological process of preeclampsia with the affection of vasoactive compounds and agents secreted by the placenta in hypertensive cases. ${ }^{5,8}$

\section{Conclusions and recommendations for future research}

Cerebroplacental ratio is a promising ultrasonographic marker for fetal and neonatal weight that could aid in prediction of neonatal wellbeing and clinical prognosis of hypertensive cases in pregnancy. Future research efforts are required to consider the integration of biomarkers secreted from the placenta to improve the detectability of neonatal wellbeing in that category of cases.

\section{Acknowledgments}

None.

\section{Funding}

None.

\section{Conflicts of interest}

The authors confirm the absence of any other conflict of interest that needs to be reported.

\section{References}

1. Shilpa K, Rupam K, Shashi BS. A prospective study of doppler analysis in pregnancy induced hypertension. IOSR Journal of Dental and Medical Sciences. 2020;19(6):53-58.

2. Bligh LN, Alsolai AA, Greer RM, et al. Prelabor screening for intrapartum fetal compromise in low-risk pregnan-cies at term: cerebroplacental ratio and placental growth factor. Ultrasound Obstet Gynecol. 2018;52(6):750 756.

3. Prior T, Mullins E, Bennett $\mathrm{P}$, et al. Prediction of intrapartum fetal compromise using the cerebroumbilical ratio: a prospective observational study. Am J Obstet Gynecol. 2013;208(124):e1-e616.

4. Bligh LN, Alsolai AA, Greer RM, et al. Cerebro-placental ratio thresholds measured within 2 weeks before birth and risk of Cesarean section for intrapartum fetal compromise and adverse neonatal outcome. Ultrasound Obstet Gynecol. 2018;52(3):340-346.

5. Khalil AA, Morales-Rosello J, Morlando M, et al. Is fetal cerebroplacental ratio an independent predictor of intrapartum fetal compromise and neonatal unit admission? Am J Obstet Gynecol. 2015;213(1):54.e1-54. e1018.

6. Morales-Roselló J, Khalil A, Morlando M, et al. Poor neonatal acid-base status in term fetuses with low cerebroplacental ratio. Ultrasound Obstet Gynecol. 2015;45(2):156-161.

7. Dunn L, Sherrell H, Kumar S. Review: systematic review of the utility of the fetal cerebroplacental ratio measured at term for the prediction of adverse perinatal outcome. Placenta. 2017;54:68-75.

8. DeVore GR. The importance of the cerebroplacental ratio in the evaluation of fetal well-being in SGA and AGA fetuses. Am J Obstet Gynecol. 2015;213:5-15.

9. Chan YH. Biostatistics102: quantitative data - parametric \& nonparametric tests. Singapore Med J. 2003;44(8):391-396.

10. Chan YH. Biostatistics 103: qualitative data -tests of independence. Singapore Med J. 2003;44(10):498-503.

11. Alanwar A, El Nour AA, El Mandooh M, et al. Prog-nostic accuracy of cerebroplacental ratio for adverse perinatal outcomes in pregnancies complicated with severe pre-eclampsia; a prospective cohort study. Pregnancy Hypertens. 2018;14:86-89. 